\title{
Parameter Effect on Tribology Performance of Biopolymer Composite Green Lubricant
}

\author{
Shih-Chen Shi ${ }^{*}$ and Jhen-Yu Wu \\ Department of Mechanical Engineering, National Cheng Kung University, \\ No. 1 University Road, Tainan 70101, Taiwan
}

(Received April 15, 2017; accepted August 4, 2017)

Keywords: loading capacity, sliding speed, transfer layer, $\mathrm{MoS}_{2}, \mathrm{HPMC}$

In this paper, the effects of loading capacity and sliding speed on the tribology properties of composite films formed by a high-concentration $\mathrm{MoS}_{2}$ additive and the biopolymer hydroxypropyl methylcellulose (HPMC) are demonstrated. The main mechanisms that affect the coefficient of friction are the real contact area and the formation of the transfer layer. Reducing the real contact area will decrease the overall coefficient of friction, while the formation of the transfer layer is a dominant factor for high-load and high-sliding-speed conditions, which results in a stable coefficient of friction. The self-lubrication phenomenon occurring after the formation of the transfer layer is mostly responsible for the excellent tribology properties of the $\mathrm{MoS}_{2} / \mathrm{HPMC}$ composite material.

\section{Introduction}

In recent years, several environmental issues have been widely discussed, including climate change anomalies, severe weather changes, lack of water resources, food shortage, and an increase in sea level. At the same time, the development of environmental technologies such as those for reduced greenhouse gas emission as well as the reduced usage and reutilization of natural resources have attracted much attention. The application of natural biopolymer materials has also recently received a lot of attention such as in electronic devices (transistors, loudspeakers, and actuators) and sensors (biosensors, gas sensors, and chromogenic sensors). The industry now requires the use of environmentally friendly or earth-friendly materials. For example, technologies substitute cutting fluid with dry plating for mechanical knives and minimum quantity lubrication (MQL) using biopolymer additives. This has been continuously developing.

In addition to dry lubrication plating and MQL, green tribological material technology has also been extensively studied and developed. ${ }^{(1,2)}$ This year happens to be the 50th anniversary of the renowned Jost Report. As pointed out in the report, friction/lubrication is not only a technical problem but also an economic problem. ${ }^{(3)}$ As a matter of fact, friction/lubrication is even more critical today: it is about the sustainability of the earth. ${ }^{(4,5)}$

Good green tribological materials have several important features in common: (1) environmentally friendly (from nature), e.g., natural oils, including soybean oil and coconut oil, and plant cellulose; ${ }^{(6,7)}$ (2) biologically decomposable; ${ }^{(8)}$ (3) widely applicable; ${ }^{(9-12)}$ (4)

${ }^{*}$ Corresponding author: e-mail: scshi@mail.ncku.edu.tw http://dx.doi.org/10.18494/SAM.2017.1658 
easy to prepare; ${ }^{(13,14)}$ and (5) reliable tribological performance ${ }^{(15-17)}$ In contrast to traditional petrochemical oil products, the lubrication performance characteristics (friction and wear reduction) of green tribological materials that meet the above requirements are not as good as those of the former. Therefore, there are still many research topics worth investigating.

In this study, a biologically and environmentally friendly biopolymer material, hydroxypropyl methylcellulose (HPMC), is introduced and its tribological performance with $\mathrm{MoS}_{2}$ additives that are known for their excellent tribological properties is strengthened. By developing this environmentally friendly lubricant and using it in sustainable manufacturing applications, the ultimate goal of this work is to reduce the use of petroleum-based lubricants and minimize damage to the earth while achieving friction reduction, wear resistance, and energy conservation.

\section{Materials and Methods}

\subsection{Preparation of $\mathrm{MoS}_{2} / \mathrm{HPMC}$ composite films and the control of film thickness}

The $\mathrm{MoS}_{2} / \mathrm{HPMC}$ composite film was produced by adding $5 \mathrm{~g}$ of HPMC to $30 \mathrm{~mL}$ of water and $130 \mathrm{~mL}$ of ethanol, followed by heating to $60{ }^{\circ} \mathrm{C}$. A MoS 2 additive $(13.5 \mathrm{~g})$ was added to the solution and ultrasonicated for $20 \mathrm{~min}$. A solution of $150 \mu \mathrm{L}$ was injected onto a silicon substrate by a micropipette. Films were observed after the solutions were left to stand for $1 \mathrm{~h}$ at $25 \pm 2{ }^{\circ} \mathrm{C}$ and $60 \pm 5 \%$ RH.

\subsection{Film analysis}

The surface morphology of the films was determined using a scanning electron microscope equipped with an energy-dispersive X-ray spectroscope (EDS).

\subsection{Tribological performance analysis of $\mathrm{MoS}_{2} / \mathrm{HPMC}$}

A pin-on-disk system was used to study the friction behavior of the composite films. Experimental details were as follows: the radius of gyration was $2 \mathrm{~mm}$, the sliding velocity was varied from 0.01 to $0.05 \mathrm{~m} / \mathrm{s}$, and a DIN 17350 chrome steel ball was used as the counter grinding ball.

\section{Results and Discussion}

The SEM cross-sectional image in Fig. 1(a) has thickness information, which shows a film thickness of approximately $100 \mu \mathrm{m}$. There are uneven portions on the surface because it is very difficult to create a clean and smooth cross section on a soft elastic film such as HPMC. In the process of splitting, the deformation and destruction of the film are likely to occur, which is indicated by the unevenness in the image. ${ }^{(18)}$ Figures $1(\mathrm{~b})-1(\mathrm{~d})$ show the results of EDS mapping, wherein the Mo and S signals (from $\mathrm{MoS}_{2}$ ) are observed from Figs. 1(b) and 1(c), whereas the $\mathrm{C}$ signal is observed in Fig. 1(d) (from HPMC). It can be seen that $\mathrm{MoS}_{2}$ particles are uniformly distributed in the HPMC substrate.

The nine-point Raman measurement method was adopted in the experiments, where nine regions with the same size were drawn on a $10 \mathrm{~mm}^{2} \mathrm{Si}$ base, followed by Raman spectral 


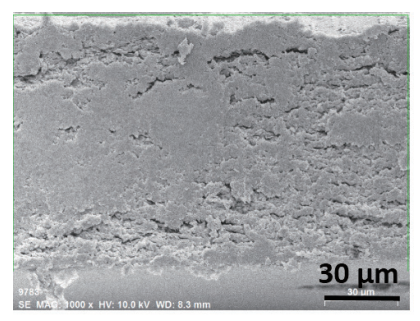

(a)

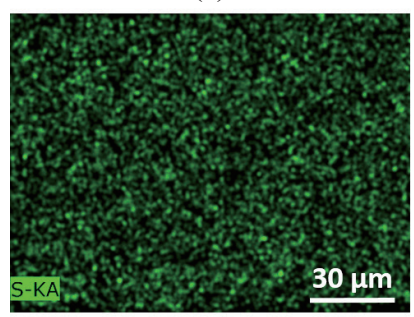

(c)

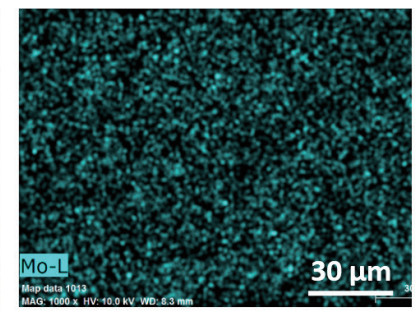

(b)

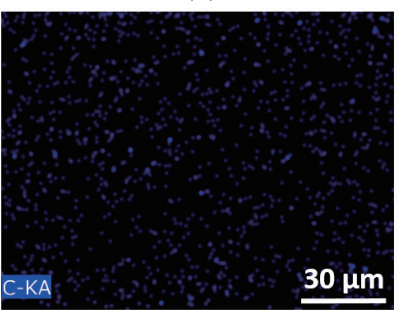

(d)

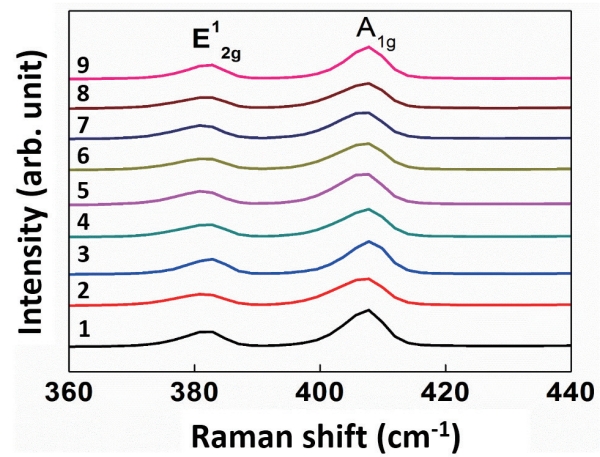

Fig. 1. (Color online) (a) SEM cross-sectional image; EDS mapping of (b) Mo, (c) S, and (d) C signals.

Fig. 2. (Color online) Additive uniformity analysis using nine-point Raman measurement.

measurement at the center of each region. The results are shown in Fig. 2. ${ }^{(5,19)}$ The distinct peaks $\mathrm{E}_{2 \mathrm{~g}}^{1}$ and $\mathrm{A}_{\mathrm{g}}^{1}$ are respectively located at 383 and $408 \mathrm{~cm}^{-1}$, corresponding to the characteristic peaks of $\mathrm{MoS}_{2}$, which indeed confirms the existence of the $\mathrm{MoS}_{2}$ additive. ${ }^{(20)}$ Further analyses of the positions and amplitudes of the nine-point peaks show that all relative errors are within $3 \%$, suggesting an excellent uniformity of the $\mathrm{MoS}_{2}$ particle distribution.

The tribological properties with $10 \%$-added $\mathrm{MoS}_{2}$ were experimentally studied under different loads, viz., 2, 5, and $8 \mathrm{~N}$. When the load was $2 \mathrm{~N}$, according to the SEM measurement, the real contact area with grinding chrome steel balls was $0.1 \mathrm{~mm}^{2}$, and the contact pressure was 20 MPa. Previous studies noted that when the load is small, even if there is an uneven deformation, in situations where the roughness of the contact surface is constant, the real contact area and the coefficient of friction are considered to be proportional to $2 / 3$ of the load. ${ }^{(21,22)}$ In this case, the coefficient of friction is related to the shearing force and hardness of the material. ${ }^{(23)}$

However, when the load is large, an insufficient load capacity will result in the elastic or plastic deformation of the film, which will increase the contact area and the overall average coefficient of friction. ${ }^{(24)}$ When there is a relative movement between two objects of distinct materials in contact, if the cohesion force of one material is smaller than the adhesion force in the contact interface, the material with a low cohesion force will break and adhere to the surface of the material with a high cohesion force. This phenomenon is called material transfer, which forms the transfer layer as a result. ${ }^{(25)}$

The condition of the transfer layer directly affects the tribological properties of the entire system. During the run-in period, a great amount of wear will be produced, but the wear will gradually decrease when the transfer layer formed (transition period) and well-covered (steady state) the object surface. ${ }^{(26)}$ After effectively adhering to or covering the objects in contact, the transfer layer material can provide a self-lubrication effect, which is one of the main mechanisms responsible for the considerable reduction in frictional resistance. ${ }^{(16,25,27)}$ 
As shown in Fig. 3, only the tribological properties during the run-in and transition periods are discussed. At a low load $(2 \mathrm{~N})$ during the run-in period, the amount of deformation of the soft film is small. The coefficient of friction is related to the shearing force of the material, and therefore it is low compared with high-load situations. However, because the transfer layer is not easy to produce, the coefficient of friction is unstable. At a high load $(5$ or $8 \mathrm{~N})$, the soft film is deformed, resulting in an increase in real contact area, which generates a higher coefficient of friction. Because the load is higher, the transfer layer is easily formed, resulting in relatively stable tribological properties.

It has been proposed in the literature that a load on the system and sliding speed will affect the generation of the transfer layer and also directly affect the subsequent tribological behavior. ${ }^{(28)}$ Figure 4 shows the tribological properties of the film at different sliding speeds. With an increase in sliding speed, the friction coefficient exhibits a stable state. This is because when the sliding speed is high, the transfer layer can easily adhere to and cover the objects in contact, producing a good self-lubrication effect.

The relationship between the average coefficient of friction in and the sliding speed obtained from Fig. 4 is plotted in Fig. 5. Here, two mechanisms are considered to affect the behavior of the coefficient of friction: the real contact area and the length of the run-in period. At a low sliding speed, because the real contact area is small, although the unstable period is relatively large (longer run-in period), the overall average coefficient of friction is lower than that at higher sliding speeds.

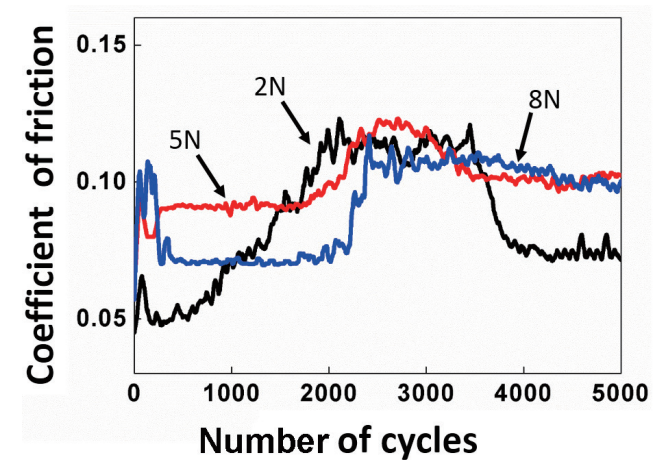

Fig. 3. (Color online) Tribological behaviors under different loads.

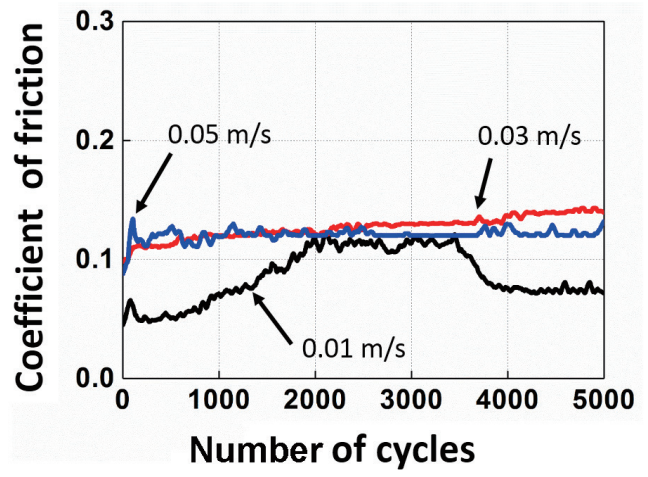

Fig. 4. (Color online) Tribological behaviors at different sliding speeds.

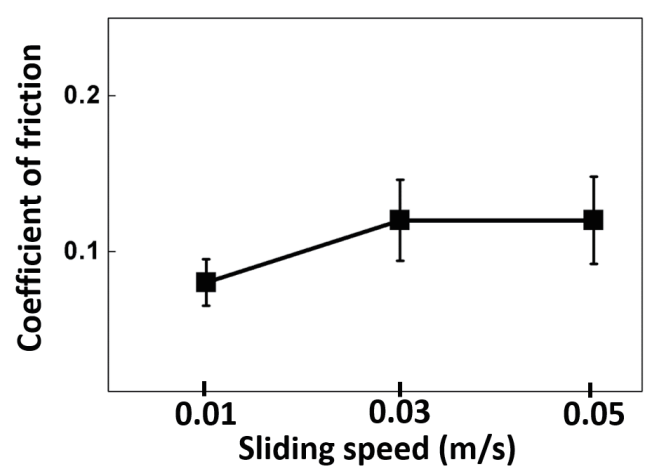

Fig. 5. Effect of sliding speed on the coefficient of friction. 
While at higher sliding speeds, because the real contact area is large, the steady state will be rapidly reached (short run-in period) and the system will have a higher coefficient of friction. This once again verifies the discussion and results shown in Fig. 3.

\section{Conclusions}

The main purpose of this research is to study the effects of a high-concentration $\mathrm{MoS}_{2}$ additive on the tribological properties of the biopolymer HPMC. The results demonstrate the importance of the growth time and uniformity of the transfer layer in the system to the tribological properties of $\mathrm{MoS}_{2} / \mathrm{HPMC}$. The real contact area of the soft film is also a very important parameter in different applications. The conclusions that may be drawn from this study are as follows:

(1) $\mathrm{MoS}_{2}$ additives and HPMC can form a completely covered and uniformly distributed composite film.

(2) An appropriate load can accelerate the formation of the transfer layer, providing a favorable coefficient of friction.

(3) The sliding speed affects the run-in period and the real contact area during wear.

\section{Acknowledgments}

The authors gratefully acknowledge the financial support for this project from the Ministry of Science and Technology in Taiwan (MOST 104-2221-E-006-057, MOST 105-2221-E-006-069, and MOST 106-2221-E-006-092-MY3). This study was supported in part by the Headquarters of University Advancement at the National Cheng Kung University, which is sponsored by the Ministry of Education, Taiwan. The authors also thank the Center for Micro/Nano Science and Technology and Instrument Center, National Cheng Kung University for technical support.

\section{References}

1 M. Nosonovsky and B. Bhushan: Green Tribology (Springer, Heidelberg, 2012) p. 3.

2 S. W. Zhang: Friction 1 (2013) 186.

3 H. P. Jost: Lubrication: Tribology; Education and Research; Report on the Present Position and Industry's Needs Working Group (HM Stationery Office, 1966).

4 S. C. Shi and T. F. Huang: Materials 10 (2017) 91.

5 S. C. Shi and F. I. Lu: Materials 9 (2016) 338.

6 N. Jayadas and K. P. Nair: J. Tribol. 128 (2006) 654.

7 S. Rani, M. Joy, and K. P. Nair: Ind. Crops Prod. 65 (2015) 328.

8 B. Thompson, P. Goldsworthy, M. Riddle, I. Snape, and J. Stark: J. Exp. Mar. Biol. Ecol. 340 (2007) 213.

9 N. Zulkifli, M. Kalam, H. Masjuki, and R. Yunus: Procedia Eng. 68 (2013) 152.

10 M. Shahabuddin, H. Masjuki, M. Kalam, M. Bhuiya, and H. Mehat: Ind. Crops Prod. 47 (2013) 323.

11 S. C. Shi and T. F. Huang: Opt. Quantum Electron. 48 (2016) 532.

12 S. C. Shi and C. C. Su: Materials 9 (2016) 612.

13 C. O. Åkerman, Y. Gaber, N. A. Ghani, M. Lämsä, and R. Hatti-Kaul: J. Mol. Catal. B: Enzym. 72 (2011) 263.

14 A. Chowdhury, D. Mitra, and D. Biswas: J. Chem. Technol. Biotechnol. 88 (2013) 139.

15 S. C. Shi, T. F. Huang, and J. Y. Wu: Materials 8 (2015) 1738.

16 S. C. Shi, J. Y. Wu, T. F. Huang, and Y. Q. Peng: Surf. Coat. Technol. 303 (2016) 250.

17 S. C. Shi: Materials 9 (2016) 856.

18 A. Jiménez, M. Fabra, P. Talens, and A. Chiralt: Carbohydr. Polym. 82 (2010) 585.

19 S. C. Shi, J. Y. Wu, and T. F. Huang: Opt. Quantum Electron. 48 (2016) 474. 
20 H. Li, Q. Zhang, C. C. R. Yap, B. K. Tay, T. H. T. Edwin, A. Olivier, and D. Baillargeat: Adv. Funct. Mater. 22 (2012) 1385.

21 N. K. Myshkin and A. V. Kovalev: Tribol. Int. 38 (2005) 910.

22 R. Bowers, W. Clinton, and W. Zisman: Ind. Eng. Chem. Chem. 46 (1954) 2416.

23 B. Bhushan: Principles and Applications of Tribology (Wiley, New York, 2013) p. 768.

24 K. Holmberg: Surf. Coat. Technol. 56 (1992) 1.

25 J. Ye, A. C. Moore, and D. L. Burris: Tribol. Lett. 59 (2015) 50.

26 J. Ye, H. Khare, and D. Burris: Wear 297 (2013) 1095.

27 R. L. Fusaro: Tribol. Int. 23 (1990) 105.

28 L. Chang, Z. Zhang, L. Ye, and K. Friedrich: Wear 262 (2007) 699.

\section{About the Authors}

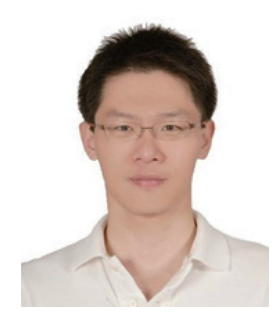

Shih-Chen Shi received his B.S. and M.S. degrees from National Cheng Kung University, Taiwan, in 1999 and 2001, respectively, and his Ph.D. degree from National Chiao Tung University, Taiwan, in 2005. From 2007 to 2012, he was the head of the R\&D Department at EVERLIGHT Electronic Co., Ltd., Taiwan. Since 2014, he has been an assistant professor at National Cheng Kung University. His research interests are in nanomaterials, tribology, LED applications, and sustainable materials.

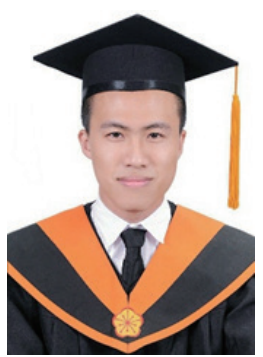

Jhen-Yu Wu received his B.S. degree from National Taipei University of Technology, and M.S. degree from National Cheng Kung University. His research interests are in nanomaterials, and tribology. 